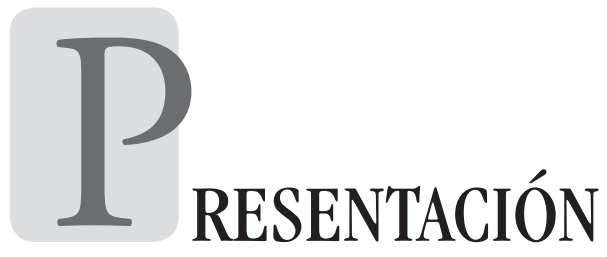

\title{
PRÓLOGO DE BUENOS AIRES
}

En la última década, los estudios de innovación han incorporado nuevas perspectivas gracias a nuevos conceptos, tales como la innovación inclusiva (Guth, 2005; Heeks, Kintu, \& Sjah, 2013; Nijhof, Fisscher, \& Looise, 2002; OECD, 2013, 2015), la innovación frugal (Basu, Banerjee, \& Sweeny, 2013; Bhatti, 2012; Rao, 2013; Zeschky, Widenmayer, \& Gassmann, 2011), la innovación social (Adams \& Hess, 2010; Cajaiba-Santana, 2014; Castro-Spila \& Unceta, 2015; European Commission, 2010, 2011, 2012, 2013), la innovación responsable (Hellström, 2003; Owen \& Goldberg, 2010; Stilgoe, Owen, \& Macnaghten, 2013; Von Schomberg, 2013) y la innovación abierta (H. Chesbrough, 2003; H. Chesbrough, 2004, 2006; O. Gassmann \& Enkel, 2004; Oliver Gassmann, Enkel, \& Chesbrough, 2010). Estas conceptualizaciones rompen con el esquema lineal de la innovación y con el ámbito exclusivo de la innovación basada en la investigación y desarrollo en el campo empresarial. Así, emerge con fuerza en los estudios de innovación una apertura 
hacia una visión distribuida del conocimiento, una pluralidad de agencias innovadoras y una concreta preocupación por la responsabilidad y por la inclusión de los más desfavorecidos.

En este contexto, el presente numero es fruto del Congreso dinámicas de la innovación: innovación social, innovación pública e innovación financiera, realizado en Buenos Aires en diciembre de 2014, organizado por la Facultad de Ciencias Económicas de la Universidad de Buenos Aires y por Sinnergiak Social Innovation de la Universidad del País Vasco.

El número incluye los trabajos presentados al congreso y seleccionados para su publicación y la Declaración de Buenos Aires, que impulsa una agenda de innovación inclusiva como un espacio de convergencia de la innovación social, pública y financiera.

En el primer artículo, Innovación social, políticas públicas y aprendizaje organizacional: el programa nacional de cuidados domiciliarios, realizado por Fassio, Rutty, Ortiz Rojas, Noriega y Aijenbon, se presentan los resultados empíricos de una investigación sobre el Programa Nacional de Cuidados Domiciliarios (PNDC) para adultos mayores y el aprendizaje organizacional producido en las organizaciones que ejecutan este programa.

Posteriormente, Sturm en la palabra innovación: teoría y práctica de caprichosas interpretaciones schumpeterianas, estudia ciertos atributos propios de la palabra innovación en tanto concepto teórico y su relevancia en torno a problemáticas relacionadas con las premisas de desarrollo en sociedades emergentes.

En el trabajo Innovación: gobernanza reflexiva y Tecnologias de la Información y las Comunicaciones (TIC) en la Gerencia Pública Argentina, su autora Gorrochategui explora cambios en el paradigma de la gestión pública y se interroga sobre si las herramientas disponibles son las adecuadas para dar solución a los problemas que requieren atención frente a demandas sociales crecientes.

Por su parte, el artículo Innovación responsable en políticas públicas: la bancarización obligatoria de las personas mayores en Argentina, elaborado por Fassio y García Fronti, analiza la problemática de la bancarización de las personas mayores, en particular el uso de tarjeta de débito en operaciones electrónicas y tiene como objetivo estudiar, desde la perspectiva de este mismo grupo etario que nunca había utilizado tarjetas de débito, si esta innovación es responsable.

Por último, Herrera explora en Bonos de impacto social: de la innovación social a la innovación financiera responsable, el desafió la innovación social requiere de nuevas políticas innovadoras. El artículo describe un instrumento financiero denominado Bonos de Impacto Social, que podría ser útil para impulsar la participación de la administración pública en el fomento de la innovación social. Siendo los bonos un instrumento financiero, analiza los debates que cobran especial relevancia sobre la responsabilidad y la irresponsabilidad en la industria de los servicios de este tipo bienes.

La publicación se cierra con la Declaración de Buenos Aires sobre innovación inclusiva, que consiste en una agenda que busca impulsar la investigación, la internacionalización y la intervención convergente en el campo de la innovación social, pública y financiera.

\section{Alfonso Unceta María Teresa Casparri Javier Castro-Spila Javier García-Fronti}

